\title{
NOTE ON AN ELEMENTARY PROBLEM OF INTERPOLATION
}

\author{
P. ERDÖS AND G. GRÜNWALD
}

The unique polynomial of degree $n-1$ assuming the values $y_{1}, y_{2}, \cdots, y_{n}$ at the abscissas $x_{1}, x_{2}, \cdots, x_{n}$, respectively, is given by the Lagrange interpolation formula

$$
L_{n}(x)=y_{1} l_{1}(x)+y_{2} l_{2}(x)+\cdots+y_{n} l_{n}(x)
$$

where

$$
l_{k}(x)=\frac{\omega(x)}{\omega^{\prime}\left(x_{k}\right)\left(x-x_{k}\right)}, \quad \quad k=1,2, \cdots, n,
$$

(fundamental polynomials of the Lagrange interpolation) and the polynomial $\omega(x)$ is defined by

$$
\omega(x)=c\left(x-x_{1}\right)\left(x-x_{2}\right) \cdots\left(x-x_{n}\right),
$$

where $c$ denotes an arbitrary constant not equal to zero.

In this note we prove the following theorem:

TheOREM. In the Lagrange interpolation formula let $x_{k}=x_{k}^{(n)}$ $=\cos (2 k-1) \pi / 2 n=\cos \theta_{k}^{(n)},(k=1,2, \cdots, n)$, which implies $\omega(x)$ $=T_{n}(x)=\cos (n \operatorname{arc} \cos x)=\cos n \theta$ (Tschebycheff polynomial). Then

$$
\left|l_{k}^{(n)}(x)\right|=\left|\frac{\omega(x)}{\omega^{\prime}\left(x_{k}\right)\left(x-x_{k}\right)}\right|<\frac{4}{\pi}, \quad-1 \leqq x \leqq+1,
$$

for all $n$ and $k$, and furthermore

$$
\lim _{n \rightarrow \infty}\left|l_{1}^{n)}(+1)\right|=\lim _{n \rightarrow \infty}\left|l_{n}^{(n)}(-1)\right|=\frac{4}{\pi} .
$$

In this connection Fejér* proved for all $n, k$, and $x,(-1 \leqq x \leqq+1)$, (6)

$$
\left|l_{k}^{(n)}(x)\right|<2^{1 / 2} .
$$

Of course (5) implies that inequality (4) is the best possible in the following sense: For any $\epsilon>0$ there exist values of $n, k$, and $x$,

${ }^{*}$ L. Fejér, Lagrangesche Interpolation und die zugehörigen konjugierten Punkte, Mathematische Annalen, vol. 106 (1932), pp. 1-55; see pp. 10,11. This paper will hereafter be referred to as $\mathrm{L}$. 
$(-1 \leqq x \leqq+1)$, such that

$$
\left|l_{k}^{(n)}(x)\right|>\frac{4}{\pi}-\epsilon .
$$

Our proof depends upon the Hermite interpolation formula which gives the unique polynomial $H(x)$ of degree $2 n-1$ satisfying the conditions

$$
H\left(x_{k}\right)=y_{k}, \quad H^{\prime}\left(x_{k}\right)=y_{k}{ }^{\prime}, \quad k=1,2, \cdots, n,
$$

where the $y_{k}$ and $y_{k}^{\prime}$ are given numbers. It is easy to show that*

$$
H(x)=\sum_{k=1}^{n} y_{k} v_{k}(x)\left\{l_{k}(x)\right\}^{2}+\sum_{k=1}^{n} y_{k}^{\prime}\left(x-x_{k}\right)\left\{l_{k}(x)\right\}^{2}
$$

where

$$
\begin{gathered}
v_{k}(x)=1-\left(x-x_{k}\right) \frac{\omega^{\prime \prime}\left(x_{k}\right)}{\omega^{\prime}\left(x_{k}\right)} \\
\sum_{k=1}^{n} v_{k}(x)\left\{l_{k}(x)\right\}^{2} \equiv 1
\end{gathered}
$$

For the Tschebycheff abscissas we have

$$
v_{k}(x)=v_{k}^{(n)}(x)=\frac{1-x x_{k}^{(n)}}{1-\left(x_{k}^{(n)}\right)^{2}}, \quad x_{k}^{(n)}=\cos (2 k-1) \pi / 2 n .
$$

Fejér proved (6) by aid of the simple inequality $v_{k}^{(n)}(x) \geqq 1 / 2$. $\dagger$

We also need the following result due to M. Riesz: $\ddagger$

Lemma. A trigonometric polynomial of degree $n-1$ assumes the maximum of its absolute value at a point whose distance from any of the roots of this trigonometric polynomial is not less than $\pi /[2(n-1)]$.

We are now in position to prove the theorem. For $n=1$ and $n=2$

$$
\begin{aligned}
\left|l_{1}^{(1)}(x)\right|=1, \quad\left|l_{1}^{(2)}(x)\right| & =\left|\frac{(\sin \pi / 4) \cos 2 \theta}{2(\cos \theta-\cos \pi / 4)}\right| \\
& =\frac{1}{2^{1 / 2}}\left|\cos \theta+\frac{1}{2^{1 / 2}}\right|<\frac{1+2^{1 / 2}}{2}<\frac{4}{\pi},
\end{aligned}
$$

* L. Fejér, Weierstrasssche Approximation, besonders durch Hermitische Interpolation, Mathematische Annalen, vol. 102 (1930), pp. 707-725.

$\dagger$ See L, p. 5.

$\ddagger$ M. Riesz, Eine trigonometrische Interpolationsformel und einige Ungleichungen für Polynome, Jahresbericht der Deutschen Mathematiker-Vereinigung, vol. 23 (1914), pp. 354-368; pp. 363-364. 


$$
\left|l_{2}^{(2)}(x)\right|<\frac{1}{2^{1 / 2}}\left|\cos \theta-\frac{1}{2^{1 / 2}}\right|<\frac{1+2^{1 / 2}}{2}<\frac{4}{\pi} .
$$

Thus we have to consider only the case $n \geqq 3$. For the Tschebycheff abscissas we have*

$$
l_{k}^{(n)}(x)=(-1)^{k+1} n^{-1} \sin \theta_{k}^{(n)} \frac{\cos n \theta}{\cos \theta-\cos \theta_{k}^{(n)}}, \quad x=\cos \theta .
$$

From (13) it follows that $l_{k}^{(n)}(\cos \theta)$ is a trigonometric polynomial of degree $n-1$. For $2 \leqq k \leqq n-1$, the roots of $l_{k}^{(n)}(\cos \theta)$ in $(0, \pi)$ are

$$
\theta_{\nu}^{(n)}=(2 \nu-1) \frac{\pi}{2 n}, \quad 1 \leqq \nu \leqq n, \nu \neq k,
$$

and since $\theta_{\nu+1}^{(n)}-\theta_{\nu}^{(n)}=\pi / n, \theta_{1}^{(n)}-0=\pi-\theta_{n}^{(n)}=\pi / 2 n,\left|l_{k}^{(n)}(\cos \theta)\right|$ assumes its maximum between $\theta_{k-1}^{(n)}$ and $\theta_{k+1}^{(n)}$. Further it is clear that $\left|l_{1}^{(n)}(\cos \theta)\right|$ and $\left|l_{n}^{(n)}(\cos \theta)\right|$ assume their maxima at $\theta=0$ and $\theta=\pi$, respectively. Let us consider first $l_{1}^{(n)}(x)$ and $l_{n}^{(n)}(x)$. According to the last remark it will be sufficient to find bounds for $\left|l_{1}^{(n)}(+1)\right|$ and $\left|l_{n}^{(n)}(-1)\right|$. From (13) we have

$$
\left|l_{1}^{n)}(+1)\right|=\left|l_{n}^{(n)}(-1)\right|=\frac{\sin \theta_{1}^{(n)}}{n\left(1-\cos \theta_{1}^{(n)}\right)}=\frac{1}{n} \cot \frac{\pi}{4 n},
$$

whence

$$
\lim _{n \rightarrow \infty}\left|l_{1}^{(n)}(+1)\right|=\lim _{n \rightarrow \infty}\left|l_{n}^{(n)}(-1)\right|=\frac{4}{\pi} .
$$

By differentiation we easily see that $x \cot x$ decreases if $x$ increases so that

$$
\left|l_{1}^{(n)}(+1)\right| \leqq\left|l_{1}^{(n+1)}(+1)\right|, \quad\left|l_{n}^{(n)}(-1)\right| \leqq\left|l_{n+1}^{(n+1)}(-1)\right| .
$$

From (15) and (16) we obtain (7), that is, the second part of the statement.

We now prove that

$$
\max _{-1 \leqq x \leqq+1}\left|l_{k}^{(n)}(x)\right|<\left|l_{1}^{(n)}(+1)\right|, \quad 2 \leqq k \leqq n-1 .
$$

By (16) it suffices to show that

$$
\max _{-1 \leqq x \leqq+1}\left|l_{k}^{(n)}(x)\right|=\left|l_{k}^{(n)}\left(\mu_{k}\right)\right|<\left|l_{1}^{(2)}(+1)\right|=\frac{1}{2}\left(1+2^{1 / 2}\right) .
$$

* See L, p. 5 . 
In order to prove (18) we show that

$$
v_{k}^{(n)}\left(\mu_{k}\right)>\frac{13}{18}, \quad 2 \leqq k \leqq n-1 .
$$

Then (11) furnishes, since $v_{k}(x) \geqq 0$,

$$
1=\sum_{k=1}^{n} v_{k}^{(n)}\left(\mu_{k}\right)\left\{l_{k}^{(n)}\left(\mu_{k}\right)\right\}^{2}>\frac{13}{18}\left\{l_{k}^{(n)}\left(\mu_{k}\right)\right\}^{2},
$$

that is,

$$
\left|l_{k}^{(n)}\left(\mu_{k}\right)\right|<\left[\frac{18}{13}\right]^{1 / 2}<(1.4)^{1 / 2}<1.2=\frac{1}{2}(1+1.4)<\frac{1}{2}\left(1+2^{1 / 2}\right) .
$$

Let $\mu_{k}=\cos \phi,(0<\phi<\pi)$. According to the lemma we have $\left|\phi-\theta_{k}^{(n)}\right|$ $<\pi / 2 n$. On account of (12) it is sufficient to prove

$$
\frac{1-\cos \theta_{k}^{(n)} \cos \left(\theta_{k}^{(n)}+\delta\right)}{1-\left(\cos \theta_{k}^{(n)}\right)^{2}} \geqq \frac{13}{18}, \quad \delta= \pm \frac{\pi}{2 n} .
$$

We can assume that $\theta_{k}^{(n)}<\pi / 2$ and $\delta=-\pi / 2 n$. If we write $\theta_{k}^{(n)}=3 \mu$, we have $\theta_{k}^{(n)}+\delta \geqq 2 \mu$ and $\cos \mu=t,\left(\frac{1}{2} \leqq t \leqq+1\right)$, hence

$$
\frac{\cos \left(\theta_{k}^{(n)}+\delta\right)-\cos \theta_{k}^{(n)}}{1-\cos \theta_{k}^{(n)}} \leqq \frac{\cos 2 \mu-\cos 3 \mu}{1-\cos 3 \mu}=\frac{4 t^{2}+2 t-1}{4 t^{2}+4 t+1} .
$$

The last fraction is $\leqq 5 / 9$ so that

$$
\frac{\cos \theta_{k}^{(n)}}{1+\cos \theta_{k}^{(n)}} \frac{\cos \left(\theta_{k}^{(n)}+\delta\right)-\cos \theta_{k}^{(n)}}{1-\cos \theta_{k}^{(n)}} \leqq \frac{5}{18},
$$

which is equivalent to (20). This completes the proof.

VICTORIA UNIVERSITY AND

BUDAPEST, HUNGARY 